\title{
Neuropsychobiology 1979;5:350-351
}

\section{Subject Index}

Adrenocortical hypofunction 27 Affective illness 1 Age 213

Aggressive behavior 129 Alpha attenuation response 210 Amitriptyline 181

Anticholinergic-neuroleptic antagonism 74 Antidepressant response prediction 266 Antiparkinsonism drugs 74 Antisocial personality 27 Apomorphine 136, 185, 222, 282 Aromatic amino acids decarboxylase inhibitors 317 Ashkenazi 50 Auditory stimulation 201 Augmenting-reducing 322 Autonomic arousal 27 Avoiddance learning 121

Behaviour 185 Benztropine 327 Bịpolar 50, 181 Body temperature 340 Brain serotonin 121,174 Brain serotonin levels 129 Brain serotonin turnover 129 Brain tryptophan 11 Bromolysergide 174 B-type fluorophore 156

Cardiovascular disease 167 Catastrophe theory 87 Cerebral lateralization 31 Cholesterol level 27 Cholinergic mechanism 74 Cholinergic mechanisms 327 Cïrcadian rhythm 11 Clinical 61 Clozapine 136 CNS 274 Cognitive function 31 , 46

<i-Amphetamine 213,22,266 DBH 309

Depressed patients 94 Depression 1,61,181,232,282 
EEG 309

EEG analysis 143

EEG and depression 143

Ego closeness-ego distance 31

Electroconvulsive shock 174

Empirical predictors 94

Endorphins 322

Envịironment 87

Epidemiology 61

Erythrocyte 1

Erythrocyte membranes 309

Factor analysis 207 Field dependence-independence 31 Flicker fusion 274 Follicle-stimulating hormone 185 Food intake 11

Genetics 332

Growth hormone 136,185

\section{Haloperidol 327}

Hemispheric asymmetry 301

Heroin addiction 294

Histocompatibility antigens (HLA) 50

HLA and schizophrenia 290

HL-A antigens 114,290

Hplocus 102

5-HT turnover rate 11

Human growth hormone 282

Hypothyroidism 274

Imipramine 185 Imipramine respones scale 94 Interhemispheric differences 143 Isolation 121

L-Dopa 185 Levodopa 282

Subject Index 
I--5-HTPloading 232 Z,--5-HTP treatment 232 Linkage 102 Liposomes 317 Lithlüum 1, 114, 160, 167,266 Lithium responders 114 Longitudinal 61 Lundby study 61 Luteinizing hormone 185

\section{Magnesium 1}

Mania 232

Manic-depressive, depressed 16

Manic-depressive illness 167

MAO 309

Memory consolidation 174

3-Methody-4-hydroxyphenylglycol 181

5-Methoxytryptamine 156

Methysergide 174

MMPI 94

Mortality 61,167

Motion sickness 31, 46

Mouse strains 121,129

Neurochemistry 87 Neuroendocrine studies 282 Neuroleptics 74

Neuropharmacological model 74 Noịse 201

One-trial passive avoidance 174 Organic 61 Orientation process 46

Paradox 46

p-Chlorophenylalanine 121 Pedigree 50

Perceptual integrative vulnerability 31 Pharmacotherapeutic process 74 Phospholipids 317 Polyuria 160 Prevention 332 Prisoner’s dilemma 46 Prolactin 136,294,317,327 Prolonged socio-environmental deprivation 129 
Prophylactic lithium 114 Prospective 61

Psychiatric electrophysiology 301 Psychomotor performance 274 Psychoneurosis 16 Psychopharmacology 332

Quantititative EEG 16

Rating scales 207 Recovery cycle 301 Retrograde amnesia 174 Reverse tolerance 213, 222

Schizoaffective 50

Schizophrenia $16,74,87,136,185,290$

Serum 5-HT level 232

Skin temperature 340

Sleep 340

Sexual differences 222

Sodium 1

Spectral analysis 20

Stereotyped behavior 213, 222

Subgroup of unipolar depression 102

Suicide 61, 167

Sulpiride 294

Systemic structural analysis of EEG 143

Testosterone 185 Therapy response 181 Thermoregulation 340 Thyroid hormone 274 TLC 156 Tremor 160 TRH 294 Tryptophan 121

Ultradian biological rhythms 340

Unipolar 50

Unipolar depression 266

VAER 322

Vigilance 274, 340

Visual evoked potential 301 